\title{
Minimum Stable Convergence Criteria for Stochastic Diffusion Search
}

\author{
D.R. Myatt, J.M. Bishop and S.J. Nasuto
}

October 13, 2003

\begin{abstract}
This letter presents an analysis of Stochastic Diffusion Search that derives the minimum acceptable match resulting in a stable convergence based on a general noise parameter. The applicability of SDS can therefore be assessed for a given problem.
\end{abstract}

\section{Introduction}

Stochastic Diffusion Search (SDS), first described in [1], is an efficient probabilitistic multi-agent global search and optimisation technique that has been applied to diverse problems such as text search [2], mobile robot self-localisation [3], object recognition [4] and site selection for wireless networks [5]. Additionally, a hybrid SDS and n-tuple RAM [6] technique has been used to track eyes in video sequences [7]. Previous analysis of SDS has investigated its convergence [8] and resource allocation [9] using Markov Chains and Ehrenfest Urn models under a variety of noise conditions. This paper will outline the derivation of a new, practical, convergence condition (underpinning that suggested in [10]) that is fully supported by appropriate numerical simulations.

\section{Stochastic Diffusion Search}

SDS employs a population of $k$ agents, each of which maintains a hypothesis in the search space, in order to locate the optima of a given objective function. 
The algorithm consists of an Initialisation Phase followed by the iteration of the Test and Diffusion Phase.

\section{Initialisation}

Typically the initial the hypothesis of each agent is selected randomly based on a uniform probability distribution over the search space. If information about probable solutions is available a priori this can be used to bias the initial selection of hypotheses.

\section{Test Function}

The Test Function returns a boolean result and is constructed domain dependently. For a given hypothesis it stochastically samples some subset of the objective function (e.g. in a pattern matching task it may test for the presence of a randomly selected pattern subfeature) and returns true is this evaluation is indicative of a 'good' hypothesis and false otherwise. The Test Score for a given hypothesis is the probability that the Test Function will return true, and is hence indicative of the true value of the objective function.

\section{Test Phase}

Each agent applies the Test Function to its current hypothesis. If the Test Function returns true the agent becomes active and otherwise becomes inactive.

\section{Diffusion Phase}

Each inactive agent, $A$, select another agent $B$ at random. If $B$ is active then the hypothesis of $B$ is copied to $A$. Conversely, if $B$ is also inactive then $A$ selects another hypothesis randomly over the entire search space.

\section{Convergence}

As iterations progress clusters of agents with the same hypothesis form. At convergence the largest cluster of agents is associated with the optimal solution.

\section{Expected cluster size formulation of SDS in homogeneous background noise}

In this section, $\alpha_{\min }$, the minimum Test Score that will provide a stable nonzero convergence will be derived. A simplifying assumption is that, by considering only the mean transition of agents between different clusters, rather than the full probability distribution (as investigated in [9]), a sufficiently ac- 
curate behavioural model of SDS may be produced. The noise model that will be assumed is that of 'homogeneous background noise', where there is a single optimal solution with Test Score $\alpha$ (that has a negligible probability of being selected) and also that every hypothesis that is non-optimal will correspond to a distractor of Test Score $\beta$. Let $\bar{c}_{i}$ be the mean number of active agents with the optimal solution as a proportion of the total population.

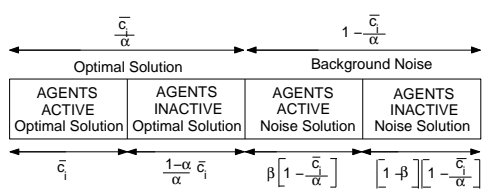

Figure 1: Illustration of the current state of the search in iteration $i$ in terms of $\bar{c}_{i}, \alpha$ and $\beta$

During the diffusion phase, the mean number of inactive agents selecting an agent within the optimal cluster is given by $g\left(\bar{c}_{i}, \alpha, \beta\right) \bar{c}_{i}$, where $g$ yields the number of inactive agents for a given iteration. From Figure $1 \mathrm{~g}$ can be immediately written as

$$
g\left(\bar{c}_{i}, \alpha, \beta\right)=\frac{1-\alpha}{\alpha} \bar{c}_{i}+(1-\beta)\left(1-\frac{\bar{c}_{i}}{\alpha}\right) .
$$

Therefore, the function $f$ that defines the transition between the size of a cluster of active agents with the optimal hypothesis, in consecutive iterations, is

$$
f\left(\bar{c}_{i}, \alpha, \beta\right)=\alpha\left(\bar{c}_{i}+g\left(\bar{c}_{i}, \alpha, \beta\right) \bar{c}_{i}\right)
$$

and consequently

$$
\bar{c}_{i+1}=\alpha\left[\bar{c}_{i}+\left(\frac{1-\alpha}{\alpha} \bar{c}_{i}+(1-\beta)\left(1-\frac{\bar{c}_{i}}{\alpha}\right)\right) \bar{c}_{i}\right] .
$$

Figure 2 considers (3.3) as a 1 dimensional iterated map, and graphically it can be seen that for an attractor to exist the condition

$$
\frac{d \bar{c}_{i+1}}{d \bar{c}_{i}}>1
$$

must hold for $\bar{c}_{i}=0$. Differentiating (3.3) wrt $\bar{c}_{i}$ yields

$$
\frac{d \bar{c}_{i+1}}{d \bar{c}_{i}}=\alpha(2-\beta)-2 \bar{c}_{i}(\alpha-\beta)
$$




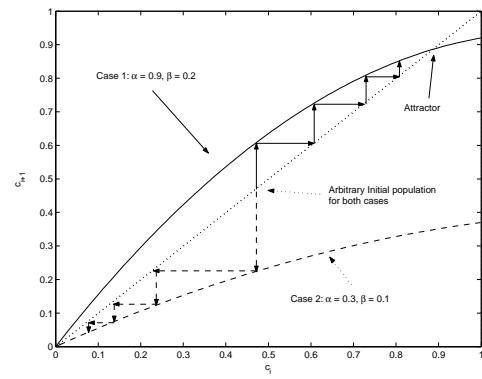

Figure 2: A 1 dimensional iterated map showing how different values of $\alpha$ and $\beta$ can induce a stable convergence.

and it follows that the minimum value of $\alpha$ for which the constraint in (3.4) holds is

$$
\alpha_{\min }=\frac{1}{2-\beta} .
$$

Hence, for any $\alpha<\alpha_{\min }$ the size of the cluster will tend to zero for any initial cluster size and the search will not converge.

\section{Numerical Results}

SDS was performed on two simulated search spaces, one with an optimal solution $\alpha=\alpha_{\min }+0.01$ and the other $\alpha=\alpha_{\min }-0.01$, while homogeneous background noise was varied between 0 and 0.99 in increments of 0.01. An initial cluster of agents with the optimal solution was seeded but the probability of an agent subsequently selecting it from the search space was zero. 10000 agents were used in each case and the cluster size of the optimal solution was measured after 1000 iterations.

Figure 3 shows that after 1000 iterations the cluster size of the search with $\alpha<\alpha_{\min }$ had returned to zero (as expected), while SDS successfully retained a cluster for $\alpha>\alpha_{\min }$. The experimental value of $\alpha_{\min }$ has therefore been shown to be \pm 0.01 of the theoretical, validating the theory. 


\section{Conclusion}

This paper has described a novel formulation for the SDS algorithm that, under some simplifying conditions, allows the calculation of the minimum match, $\alpha_{\min }$ in a given search space that will guarantee SDS to stably converge. In practical situations when these conditions are violated this value still provides a useful estimation.

\section{References}

[1] J.M. Bishop.: 'Stochastic Searching Networks'. Proc. 1st IEE Conf. on Artifical Neural Networks, 1989, (London), pp. 329-331

[2] S.J. Nasuto.: 'Resource allocation of the Stochastic Diffusion Search'. PhD Thesis, 1999, (University of Reading)

[3] N.I. Katevas, N.M. Sgouros, S.G. Tzafestas, G. Papakonstantinou, P. Beattie, J.M. Bishop, P. Tsanakas, D. Koutsouris.: 'The Autonomous Mobile Robot SENARIO: A Sensor-Aided Intelligent Navigation System for Powered Wheelchairs'. IEEE Robotics and Automation 4(4) 1997, pp. 60-70

[4] H.J.Grech-Cini, G.T.McKee.: 'Locating the Mouth Region in Images of Human Faces'. In P.S.Schenker (Ed.), Proceedings of SPIE - The International Society for Optical Engineering, Sensor Fusion VI 2059, (Massachusetts), 1993

[5] R.M. Whitaker, S. Hurley.: 'An agent based approach to site selection for wireless networks'. Proc ACM Symposium on Applied Computing (Madrid), March 2002, pp. 574-577

[6] I. Aleksander and T. J. Stonham. 'A guide to pattern recognition using random-access memories'. IEE Proceedings-E Computers and Digital Techniques 2(1), 1979, pp. 29-40.

[7] J.M. Bishop, P. Torr.: 'The Stochastic Search Network'. In R. Linggard, D.J. Myers, C. Nightingale (Eds.), Neural Networks for Images, Speech and Natural Language, 1992, New York, Chapman and Hall 
[8] S.J. Nasuto, J.M. Bishop.: 'Convergence Analysis of Stochastic Diffusion Search'. Journal of Parallel Algorithms and Applications 14:2, 1999, pp. 89-107

[9] S.J. Nasuto, J.M. Bishop, S. Lauria.: 'Time Complexity of Stochastic Diffusion Search'. Neural Computation '98, 1998, (Vienna)

[10] H.J. Grech-Cini.: 'Locating Facial Features'. PhD Thesis, 1995, (University of Reading) 


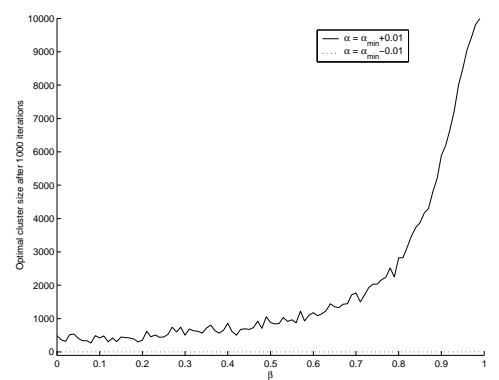

Figure 3: A graph showing the cluster size after 1000 iterations for values of $\alpha=\alpha_{\min } \pm 0.01$. 